\title{
The Parkes methanol multibeam survey
}

\author{
R. J. Cohen ${ }^{1}$, J. L. Caswell ${ }^{2}$, K. Brooks ${ }^{2}$, M. G. Burton ${ }^{3}$, \\ A. Chrysostomou ${ }^{4}$, J. Cox $^{9}$, P. J. Diamond ${ }^{1}$, S. Ellingsen ${ }^{5}$, \\ G. A. Fuller ${ }^{6}$, M. D. Gray ${ }^{6}$, J. A. Green ${ }^{1}$, M. G. Hoare ${ }^{7}$, \\ M. R. W. Masheder ${ }^{8}$, N. McClure-Griffiths ${ }^{2}$, M. Pestalozzi $^{4}$, \\ C. Phillips ${ }^{2}$, M. Thompson ${ }^{4}$, M. Voronkov ${ }^{2}$, A. Walsh ${ }^{3}$, \\ D. Ward-Thompson ${ }^{9}$, D. Wong-McSweeney ${ }^{6}$ and J. A. Yates ${ }^{10}$

\footnotetext{
${ }^{1}$ Jodrell Bank Observatory, The University of Manchester; ${ }^{2}$ Australia Telescope National

${ }^{6}$ University of Tasmania; ${ }^{7}$ The University of Manchester; ${ }^{8}$ University of Leeds; ${ }^{9}$ Bristol University; ${ }^{10}$ University College, London.
} \\ Facility; ${ }^{3}$ University of New South Wales; ${ }^{4}$ University of Hertfordshire; ${ }^{5}$ University of Cardiff;

\begin{abstract}
A new 7-beam methanol multibeam receiver was successfully commissioned at Parkes Observatory in January 2006, and has begun surveying the Milky Way for newly forming massive stars, that are pinpointed by strong methanol maser emission at $6.7 \mathrm{GHz}$. The receiver was jointly constructed by Jodrell Bank Observatory and the Australia Telescope National Facility for use on the Parkes and Lovell Telescopes. The whole galactic plane is being surveyed within latitudes $\pm 2^{\circ}$, with a velocity resolution of $0.1 \mathrm{~km} \mathrm{~s}^{-1}$ and a $5-\sigma$ sensitivity of $\sim 0.7 \mathrm{Jy}$. Altogether 200 days of observing will be required.
\end{abstract}

Keywords. masers, surveys, stars: formation, ISM: molecules, radio lines: ISM

The Parkes Methanol Multibeam Survey is the most sensitive survey yet undertaken for massive young stars in the Galaxy, and will provide the first unbiased catalogue of these objects. The first 26 days of observations have yielded 377 methanol sources, of which 150 are new discoveries. Most of the new sources are weak ( $\leqslant 4 \mathrm{Jy})$. The radial velocities show that the survey is detecting masers on the far side of the Galaxy, outside the solar circle. The most distant source found so far is $13.7 \mathrm{kpc}$ from the galactic centre and $19.6 \mathrm{kpc}$ from the Sun. Accurate ( 0.1 arcsecond) positions for the masers are being measured with the ATCA and with MERLIN, and cross-correlated against other galactic surveys including MSX, IRAS, Spitzer/GLIMPSE and UKIRT/UKIDSS, to identify counterparts at other wavelengths. The Parkes survey is expected to take 100 days, after which the receiver will be moved to Jodrell Bank to complete the survey of the northern galactic plane.

The receiver covers the frequency range 6.0-6.7 GHz, delivering two $300 \mathrm{MHz}$ IF bands in dual circular polarization. The telescope is scanned in galactic longitude, with data read out every 5 seconds. Spectra cover a $4 \mathrm{MHz}$ band, corresponding to a velocity range of $180 \mathrm{~km} \mathrm{~s}^{-1}$ (2048 channels, $0.09 \mathrm{~km} \mathrm{~s}^{-1}$ resolution). Regions towards the galactic centre are scanned more than once with different velocity settings to cover all likely radial velocities for the maser emission. A second frequency band, centred on the $6035 \mathrm{MHz}$ line of $\mathrm{OH}$. is observed in parallel with the methanol. Detection using the multibeam provides a position accurate to $\sim 20^{\prime \prime}$, with follow-up interferometry able to increase the precision to $\sim 0.1^{\prime \prime}$.

The survey webpage has further information and updates:

http://www.manchester.ac.uk/jodrellbank/research/methanol 